Research Article

\title{
Mechanism Analysis on Hydraulic Fracture Initiation under the Influence of Induced Stress in Shale Gas
}

\author{
Shanyong Liu, Yishan Lou ${ }^{1}$, Di Chen ${ }^{2}$, Yafei, $\mathrm{Ni}^{1}$, Cheng $\mathrm{Ni}^{1}$ and Huachang Wen ${ }^{3}$ \\ ${ }^{1}$ School of Petroleum Engineering, Yangtze University, Wuhan 430100, China \\ ${ }^{2}$ School of Energy Resources, China University of Geosciences, Beijing 100083, China \\ ${ }^{3}$ UMBC union, 12 Avenue Ouin, Toulouse 0254, France
}

Received 1 June 2016; Accepted 29 July 2016

\begin{abstract}
To study the fracture network propagation mechanism in shale gas reservoirs and determine the influence of induced stress from the growth of multiple fractures, this paper describes the crack initiation pattern in a shale reservoir based on a core laboratory experiment and volume fracturing concept. In accordance with linear elastic fracture mechanics and based on the mathematical model of induced stress field with single fracture as reference, the rock surrounding stress equation of multiple fractures in a horizontal well was deduced. Prediction models of fracture pressure under different initiation patterns were established. Induced stress correction factor was proposed to simplify and correct the prediction models. Results demonstrate that the mechanical parameters of rock directly affect the fracture initiation pattern in shale reservoirs. Tensile failure on the bedding surface primarily occurs, along with shear slippage damage for brittle rocks, whereas shear was mainly observed in plastic rocks. The morphology and distribution of fractures are closely related to induced stress field. Simulation results show that induced stress is positively correlated with fracture height and negatively correlated with fracture interval. Dimensionless fracture interval between one to two is the "gold window" to create a fracture network in fracturing design. Minimum induced stress occurs at $30^{\circ}$ and $150^{\circ}$ with the minimum horizontal stress direction. The study significantly contributes in the research of crack initiation law and optimization of fracture design.
\end{abstract}

Keywords: Hydraulic Fracture Initiation, Induced Stress, Initiation Pattern, Fracture Propagation Mechanism

\section{Introduction}

Shale gas exploration in China is rapidly developing, and multi-stage fracturing technology revolutionized this field [1]. However, studies on shale gas reservoirs indicate that subsurface conditions are complex in new blocks. The substantial depth, complex geological background, evident variance of rock constituents, characteristics of the mechanical parameters of rock, and crustal stress in different zones cause difficulties in fracturing design and field operation [2], [3]. Research on how a rock constitutive model affects the mechanism of fracture propagation is lacking. Induced stress caused by open cracks is the key factor that affects fracture growth and the formation of a fracture network. The coupling of in-situ stress and induced stress also causes difficulties. Hence, developing a precise fracturing model is crucial to optimize technical parameters and enhance stimulation effects in shale gas reservoir exploration.

The spatial distribution and propagation mechanism of hydraulic fractures was analyzed in this paper by employing a core test in a laboratory and conducting theoretical analysis. The initiation patterns with different features of rock mechanics were discussed from the meso-mechanic perspective. Based on elastic constitutive theory, the principle of superimposed stress was applied in developing

*E-mail address: liushanyong2012@163.com

ISSN: 1791-2377 @ 2016 Eastern Macedonia and Thrace Institute of Technology. All rights reserved. the circumferential stress analytical model.

\section{State of the art}

Hydraulic fracturing technology is based on linear elastic fracture mechanics in classical theory. Artificial fracture is hypothesized to be an open mode and shaped as a symmetrical bi-wing plane fissure. Fracture toughness equation is a criterion for fracture extension [4]. However, micro-seismic monitoring results determined that hydraulic fractures (HF) develop like a complex fracture network in a plane and lengthwise but not in a symmetrical shape [5]. Shearing slippage damage of nature fractures (NF) tend to occur when NF interacts with HF. Differential principle stress, approaching angle, and operation pressure were the main factors that cause this damage [6]. Renshaw and Pollard [7] observed that fluids penetrate towards the fracture surface initially and propagate along the maximum horizontal stress direction when the influence of discontinuities space on HF propagation was studied. And the criterion when HF orthogonally crosses NF was also provided. Gu and Weng [8] expanded the R-P criterion and included the non-orthogonal condition. By embedding planar glass discontinuities into a cast hydrostone block as proxies for cemented natural fractures, Olson proposed that HF-NF interaction occurs in three forms: 1) HF bypassing the NF by propagating around it, 2) HF arresting into the natural fracture and then diverting along it, and 3) a combination of bypass and diversion [9], [15]. Renard 
analyzed the morphology of HF propagation in a 3-D space with X-ray computed synchrotron microtomography. The results demonstrated that both hard structure (such as granules) and weak structure (such as pores and particulates) affect the HF extension pathway [10]. Xu [11], [12], [13] and Meyer and Bazan [14] developed a new mathematical model to characterize and predict the growth of induced hydraulic fracture networks in naturally fractured formations. This model consists of two perpendicular sets of vertical planar fractures that mechanically interact and considers the presence of injected fluid. Mathematical models and laboratory experiments were popular methods in analyzing fracture growth, but both approaches were studied separately and studies on the effect of the constitutive properties of rocks were lacking. Thus, this paper combines laboratory testing with numerical simulation to develop a fracturing model that can be widely used.

The remainder of this paper is organized as follows: Section 3 presents how cracks occur in shale reservoirs and establishes the prediction model of fracture pressure under the effect of multiple fractures by induced stress field. Section 4 analyzes the significance of the technical parameters of the model and discusses the stress distribution around fractures through numerical simulation. Section 5 summarizes the conclusions.

\section{Methodology}

The mechanical property of shale is quite different from that of conventional reservoirs because of its special bedding and the development degree of NF. The anisotropy mechanical properties and failure damage characteristics of the Long $\mathrm{Ma}$ $\mathrm{Xi}$ Shale Formation in $\mathrm{X}$ block were studied through laboratory experiments. The experiments showed that the mechanical parameters and fracture failure characteristics of shale changed significantly and exhibited strong anisotropy because of the different geological properties of various reservoir sections. The results of the mechanical testing analysis of the core in various zones in the X-1 well of Block $X$ were selected to comparatively examine the mechanical properties of the different layers of the shale reservoir.

\subsection{Uniaxial compression test of rock mechanics}

TAW - 2000 computer-controlled servo triaxial testing machine was used in this test (Figure 1) to test normal and high temperature and pressure, statics, dynamics, and uniaxial and triaxial compression. Uniaxial compressive strength, elastic modulus, and Poisson's ratio can be determined by employing uniaxial compression test. The initiation mode can also be determined through fracture morphology. The samples of No.1 sublayer indicate that the mean value of uniaxial compressive strength is $239.9 \mathrm{MPa}$, the elastic modulus is $30.2 \mathrm{MPa}$, and Poisson's ratio is 0.20 . These results indicate brittle characteristics with relatively high compressive strength, relatively high elastic modulus, and relatively low Poisson's ratio. The initiation mode of the samples is tensile failure along the dense lamellation plane accompanied by shear slippage damage (Figs. $2 \& 3$ ).

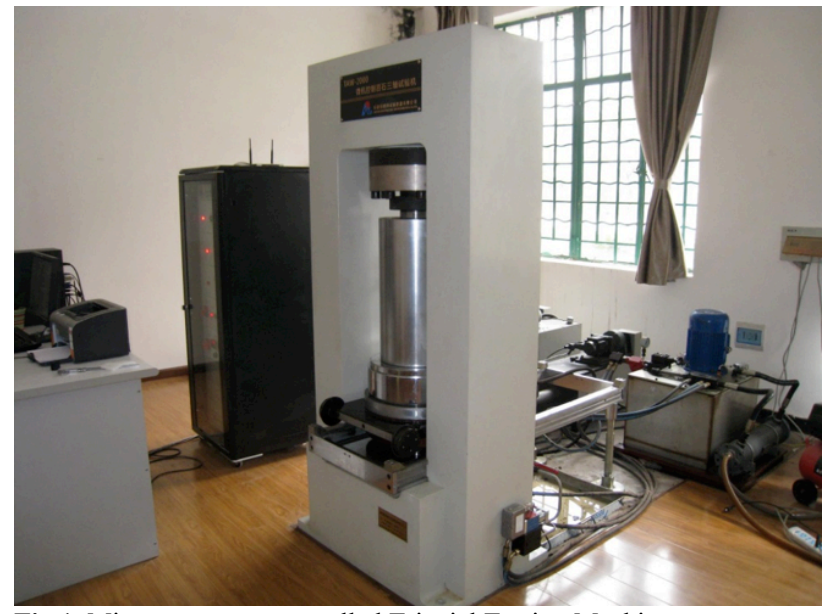

Fig.1. Microcomputer-controlled Triaxial Testing Machine

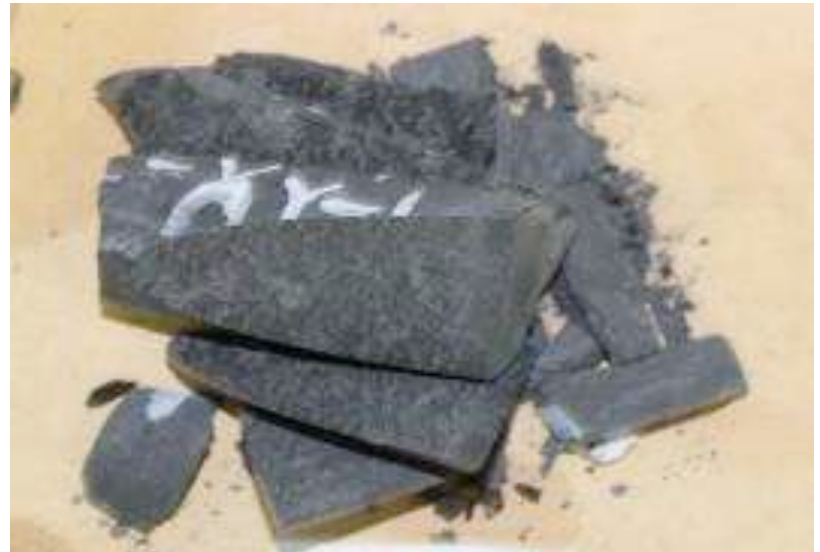

Fig.2. Morphology of No.1 sublayer uniaxial test after compression

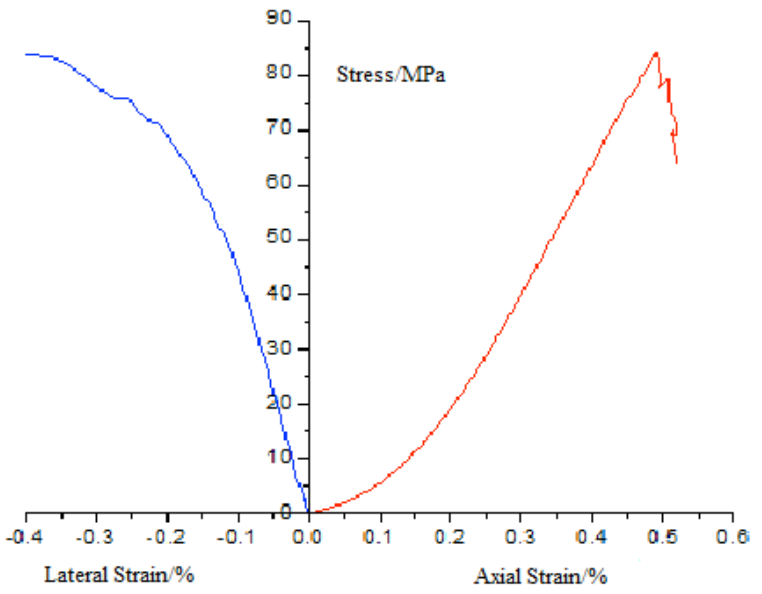

Fig.3. Stress - strain curve of No.1 sublayer

The samples of No.2 sublayer indicate high elastic modulus and low Poisson's ratio. The mean value of uniaxial compressive strength is $247.5 \mathrm{MPa}$, the elastic modulus is 27.9 MPa, and Poisson's ratio is 0.22 . These results denote hard and brittle characteristics with relatively high compressive strength, relatively high elastic modulus, and relatively low Poisson's ratio. The initiation mode of the samples is tensile failure along the dense lamellation plane accompanied by shear slippage damage (Figs. 4 \& 5) 


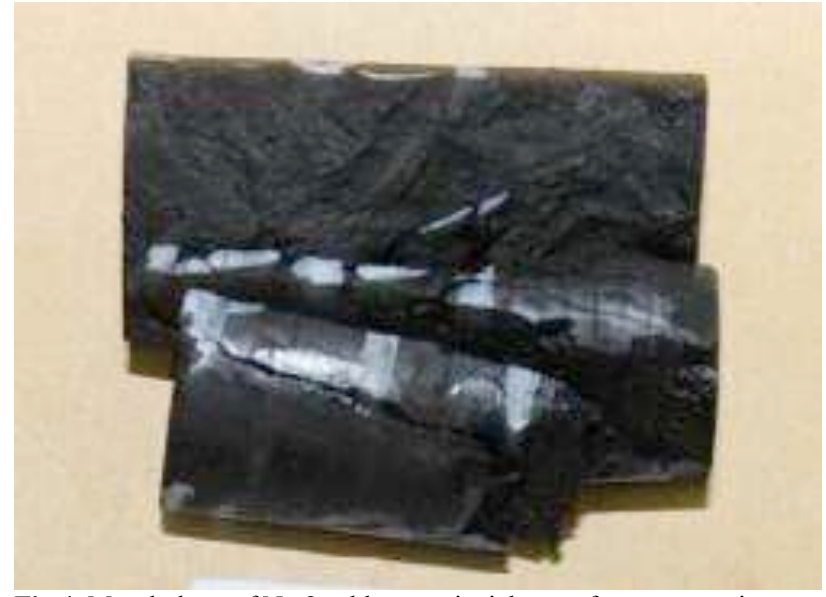

Fig.4. Morphology of No.2 sublayer uniaxial test after compression

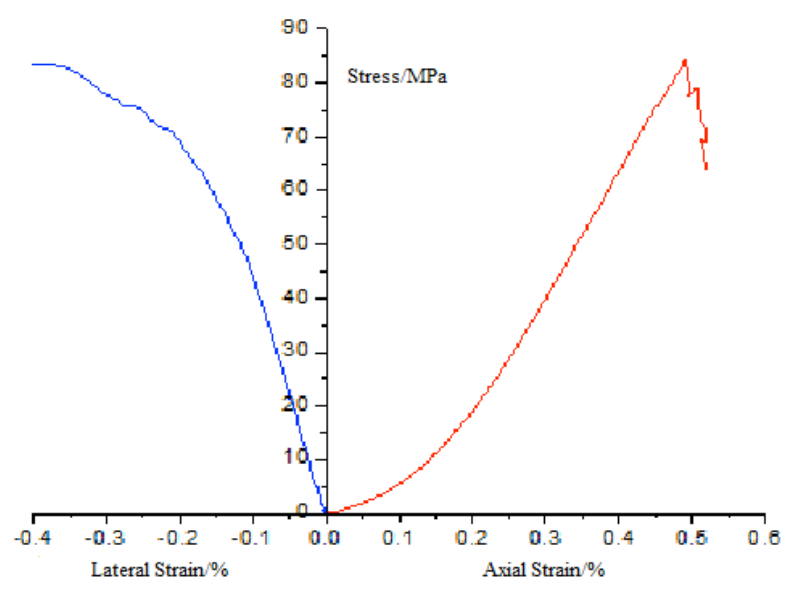

Fig.5. Stress - strain curve of No.2 sublayer

The samples of No.3 sublayer show moderate elastic modulus and moderate Poisson's ratio. The mean value of uniaxial compressive strength is $190.9 \mathrm{MPa}$, the elastic modulus is $24.8 \mathrm{MPa}$, and Poisson's ratio is 0.21 . The results indicate moderate compressive strength, moderate elastic modulus, and relatively low Poisson's ratio. The initiation mode of the samples is shear damage along the dense lamellation plane accompanied by open mode (Figs. $6 \&$ 7).

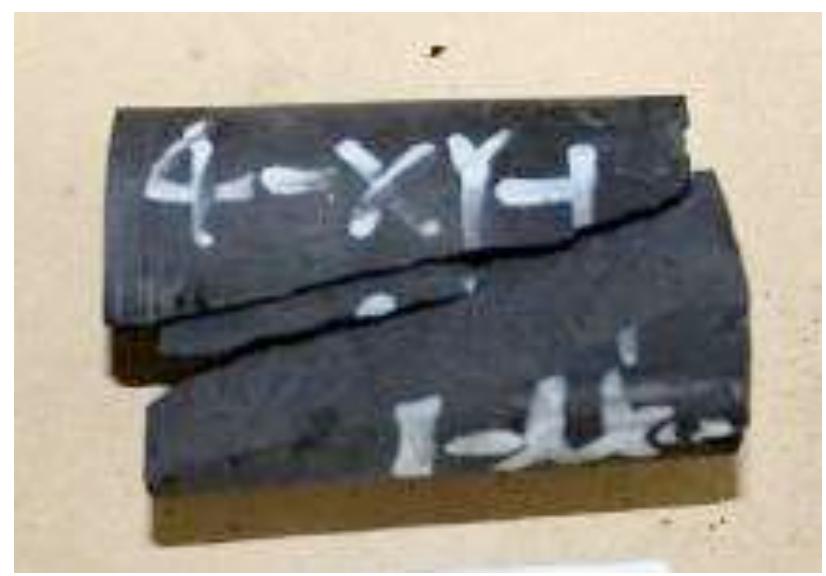

Fig.6. Morphology of No.3 sublayer uniaxial test after compression

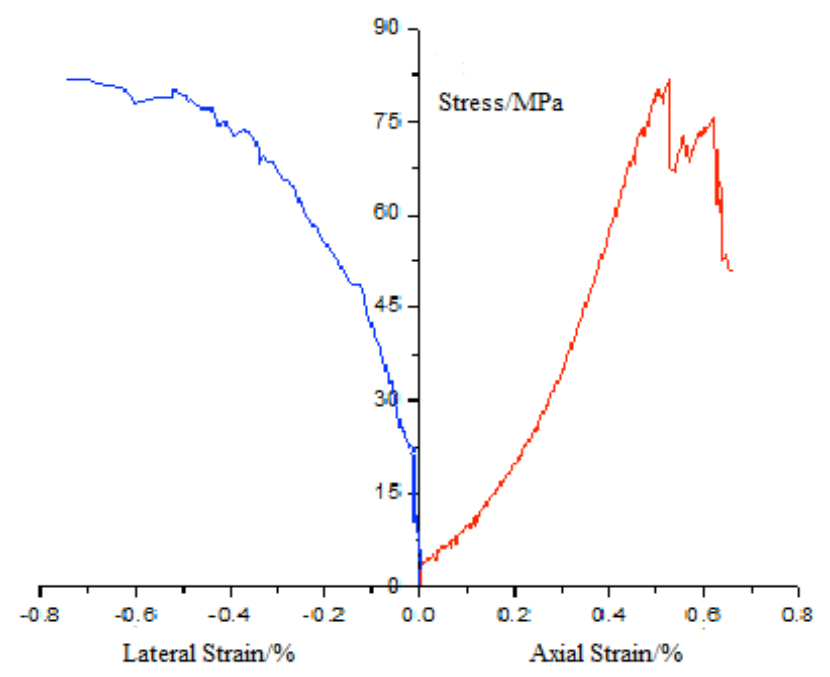

Fig.7. Stress - strain curve of No.3 sublayer

The samples of No.4 sublayer indicate moderate elastic modulus and relatively low Poisson's ratio. The mean value of uniaxial compressive strength is $219.5 \mathrm{MPa}$, the elastic modulus is $25 \mathrm{MPa}$, and Poisson's ratio is 0.23 . The results denote moderate compressive strength, moderate elastic modulus, and relatively low Poisson's ratio. The initiation mode of the samples is shear damage along dense lamellation plane accompanied by open mode (Figs. $8 \& 9$ ).

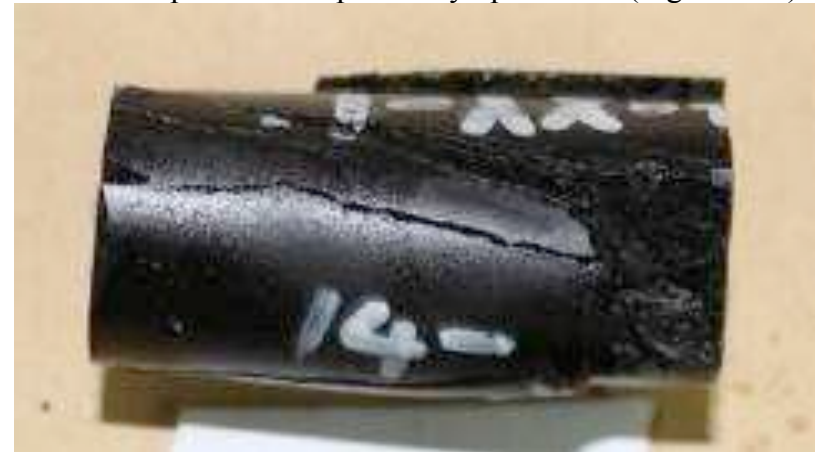

Fig.8. Morphology of No.4 sublayer uniaxial test after compression

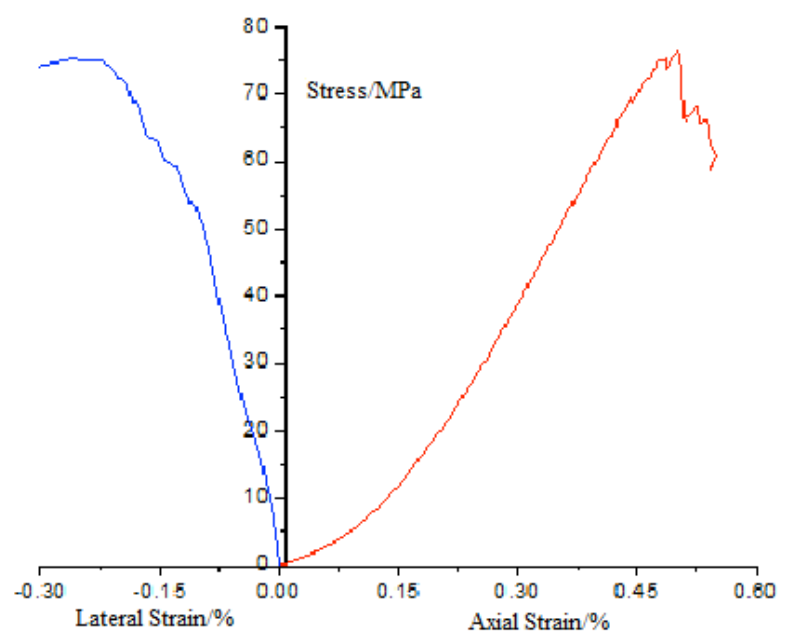

Fig.9. Stress - strain curve of No.4 sublayer

The samples of No.5 sublayer show relatively low elastic modulus and relatively high Poisson's ratio. The mean value of uniaxial compressive strength is $206.2 \mathrm{MPa}$, the elastic 
modulus is $24.4 \mathrm{MPa}$, and Poisson's ratio is 0.24 . The results indicate relatively low compressive strength, relatively low elastic modulus, and relatively high Poisson's ratio. The initiation mode of the samples shows a single shear rupture plane (Figs. $10 \& 11$ ).

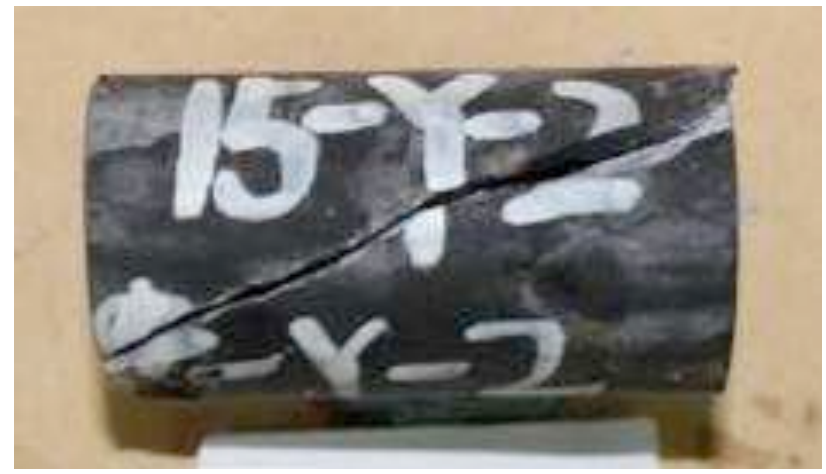

Fig.10. Morphology of No.5 sublayer uniaxial test after compression

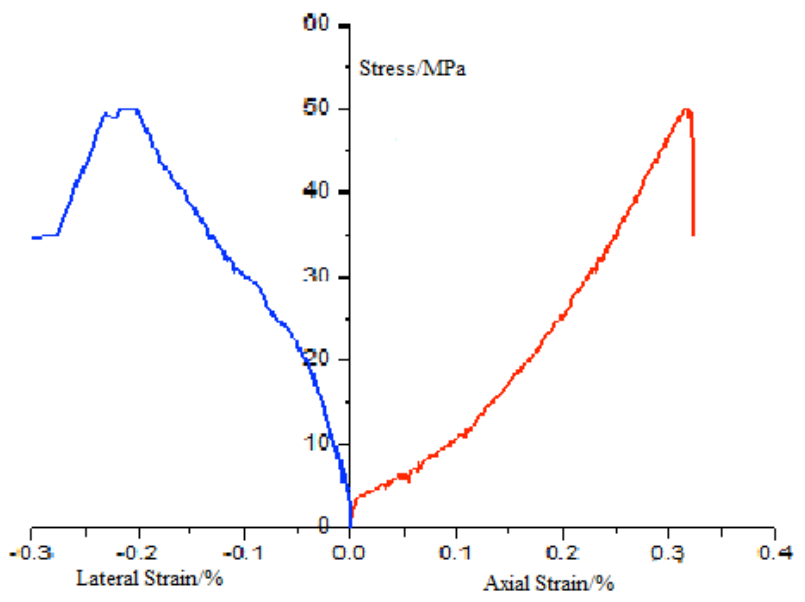

Fig.11. Stress - strain curve of No.5 sublayer

\subsection{The determination of hydraulic fracture initiating criterion}

The experimental results in Section 3.1 indicate that the fracturing mode of shale is associated with the constitutive characteristics of the rock, and different elastic modulus and Poisson ratios exhibit varying fracturing patterns. Two main initiation patterns were observed: 1) tensile failure (dominated)-shear failure; 2) shear failure (dominated)tensile failure.

\subsubsection{Tension failure criterion occurring within the rock itself of hydraulic fracture}

Fracture pressure is closely related to crustal stress. The circumferential stress that the rock is subjected to exceeds the rock's strength of extension because of the overlarge density of the liquid in the well, that is:

$$
\sigma_{\theta}=-S_{t}
$$

In the formula, $\sigma_{\theta}$ is circumferential stress, $\mathrm{MPa}$, and $\mathrm{S}_{\mathrm{t}}$ is the rock's tensile strength, MPa.

\subsubsection{Shear failure criterion of hydraulic fracture along} the natural fracture

Suppose that NF exists in the primary development zone, its development and approaching angle remain constant. Weak surface mode can be used to study the shear failure of HF along with NF. For naturally fractured formation, weak plane cohesion is zero. Thus, the fracture criterion [16] of $\mathrm{HF}$ along with NF is:

$$
\sigma_{1}-\sigma_{3}=\frac{2 \mu_{\omega} \sigma_{3}}{\left(1-\mu_{\omega} \operatorname{ctg} \beta_{2}\right) \sin 2 \beta_{2}}
$$

In the formula, $\sigma_{1}$ and $\sigma_{3}$ represent the maximum and minimum principal stress, $\mathrm{MPa}, \mu_{\mathrm{w}}$ denotes the internal friction coefficient of plane of weakness, and $\beta_{2}$ is the angle between normal of weakness plane and $\sigma_{1}$.

\subsection{Fracture pressure prediction model under multiple fractures induced stress}

With the constant improvement of stimulation technology in the horizontal well, the scale number and stage quantity in multi-staged fracturing increased. The interference effect among multiple fractures changed the stress field of the original wall rock [17],[18]. Thus, examining the mechanism of induced stress, establishing an induced stress field mathematical model, and analyzing the influencing factors are crucial in investigating the fracture initiation regularity of shale reservoirs in a horizontal well. By considering the effect of multi-fracture induced stress on the pressure system and based on the pressure balance,

$$
\begin{aligned}
& P_{E}=P_{w}-P_{p i p e}-\Delta P_{i} \\
& P_{n e t}=P_{E}-P_{c}-\sigma_{\varepsilon}
\end{aligned}
$$

In the formula, $\mathrm{P}_{\mathrm{w}}$ is bottom hole flowing pressure, $\mathrm{MPa}$, $\mathrm{P}_{\mathrm{E}}$ is fracture propagation pressure, $\mathrm{MPa}, \mathrm{P}_{\text {pipe }}$ is pipeline friction pressure, $\mathrm{MPa}, \Delta \mathrm{P}_{\mathrm{i}}$ is perforation friction pressure, $\mathrm{MPa}, \mathrm{P}_{\mathrm{c}}$ is closure pressure, $\mathrm{MPa}, \sigma_{\xi}$ is induced stress, $\mathrm{MPa}$, and $\mathrm{P}_{\text {net }}$ is net pressure in fracture, $\mathrm{MPa}$.

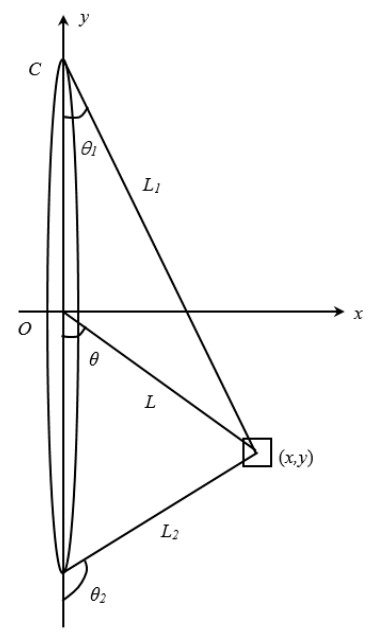

Fig.12. Vertical Fracture Schematic

Figure 12 shows that the induced stress field model can be simplified into a plane strain model [19]. According to linear elasticity mechanics, when fracture is open, the induced stress at an arbitrary point [4], [20] is:

$$
\Delta \sigma_{x}=p\left\{\frac{r}{\sqrt{r_{1} r_{2}}} \cos \left(\theta-\frac{\theta_{1}+\theta_{2}}{2}\right)+\frac{c^{2} r}{\sqrt{\left(r_{1} r_{2}\right)^{3}}} \sin \theta \sin \left[\frac{3}{2}\left(\theta_{1}+\theta_{2}\right)\right]-1\right\}
$$




$$
\Delta \sigma_{z}=p\left\{\frac{r}{\sqrt{r_{1} r_{2}}} \cos \left(\theta-\frac{\theta_{1}+\theta_{2}}{2}\right)-\frac{c^{2} r}{\sqrt{\left(r_{1} r_{2}\right)^{3}}} \sin \theta \sin \left[\frac{3}{2}\left(\theta_{1}+\theta_{2}\right)\right]-1\right\}
$$

Based on the generalized Hooke's law, the induced stress on $\mathrm{y}$ axis is:

$$
\Delta \sigma_{y}=v\left(\Delta \sigma_{x}+\Delta \sigma_{z}\right)
$$

The mathematical relationships of the remaining parameters can be described as follows:

$$
\begin{aligned}
& \left\{\begin{array}{l}
r=\sqrt{x^{2}+y^{2}} \\
r_{1}=\sqrt{x^{2}+(z+c)^{2}} \\
r_{2}=\sqrt{x^{2}+(z-c)^{2}}
\end{array}\right. \\
& \left\{\begin{array}{l}
\theta=\tan ^{-1}\left(-\frac{x}{z}\right) \\
\theta_{1}=\tan ^{-1}\left(-\frac{x}{z+c}\right) \\
\theta_{2}=\tan ^{-1}\left(-\frac{x}{z-c}\right)
\end{array}\right.
\end{aligned}
$$

In the formula, $\mathrm{P}$ is the net pressure over the fracture surface, $\mathrm{MPa}$, and $\mathrm{c}$ is semi-fracture height, m. For $\mathrm{n}$ cracks, the induced stress produced by each cracks disturbs the original stress field, and the induced stress loading on the minimum horizontal stress direction at the stress superposition area is

$$
\Delta \sigma_{x T}=\Delta \sigma_{x 1}+\Delta \sigma_{x 2}+\cdots+\Delta \sigma_{x n}
$$

By integrating Formula (10) in Formula (4), the pressure should increase for a multi-fracture stress field if each fracture must be stimulated with the same scale. Induced stress correction factor (ISCF) must be introduced.

$$
P_{n e t}{ }^{\prime}=P_{n e t}(1+\phi)
$$

Formula (5) shows that induced stress is the function of c, $\mathrm{r}$, and $\theta$. In the actual fracturing process, the influence on principal stress that is generated by induced stress of various fractures should be considered. Thus, induced stress is the function of fracture height and fracture interval. By defining $\mathrm{h} / \mathrm{d}$ as a dimensionless fracture space, the mathematical description of the ISCF obtained by regression is:

$$
\phi=1-\left(1+\left(\frac{h}{2 d}\right)^{2}\right)^{-3 / 2}
$$

In the formula, $\mathrm{h}$ is fracture height, $\mathrm{m}$, and $\mathrm{d}$ is fracture interval, $\mathrm{m}$. At the end, the fracture pressure with different fracturing initiating modes can be obtained by combining Formulas (1), (2), (3), (4), and (11).

\section{Results and discussions}

During the fracture growth process in shale gas reservoirs, stress distribution, stratum mechanical properties, properties of fracturing fluid loss, and pumping mechanisms are the main elements of fracture initiation and geometry. Therefore, determining the stress state of wall rock is crucial when the initiation and propagation law of a hydraulic fracture is analyzed.

\subsection{Relationship between induced stress and fracture} geometry parameters and laying patterns

Section III discussed that induced stress can be simplified into a function of fracture height and fracture interval. Combined with the data on the field, the relationship of induced stress loading in the minimum principal stress directions and fracture height and fracture space were obtained. Figure 13 shows that when fracture interval is constant, induced stress will increase with fracture height because the stimulated volume in lengthways elevates. Two issues should be considered for shale gas reservoirs: a) The volume of the fracture positively influences induced stress. Thus, the relationship between operation scale and induced stress should be considered in optimizing fracturing design; b) the extension in other fractures will be hindered if fracture height was uncontrolled at one crack in the fracturing treatment.

When fracture geometry is constant, induced stress decreases as fracture interval increases (Fig. 14). Fracture interval is an effective way to adjust the interference among fractures. Hence, when increasing the complexity of the fracture through fracture interaction is necessary, fracture interval must be reduced. When decreasing the complexity of the fracture is needed through fracture interaction, the fracture interval must be enlarged. When several fracture initiations occur simultaneously, the fractures will interfere with each other, which influence fracture propagation in next stage. The growth situation of two to five fractures were simulated, and the changes of induced stress can be characterized by IDCF. Figure 15 shows that the fractures interfere with each other during their propagation process. Interference is more evident if more fractures exist. With the interference of the fractures, the variation coefficient of horizontal principal stress reduces, which facilitates the formation of a complex fracture network. Thus, laying patterns are crucial in fracturing design.

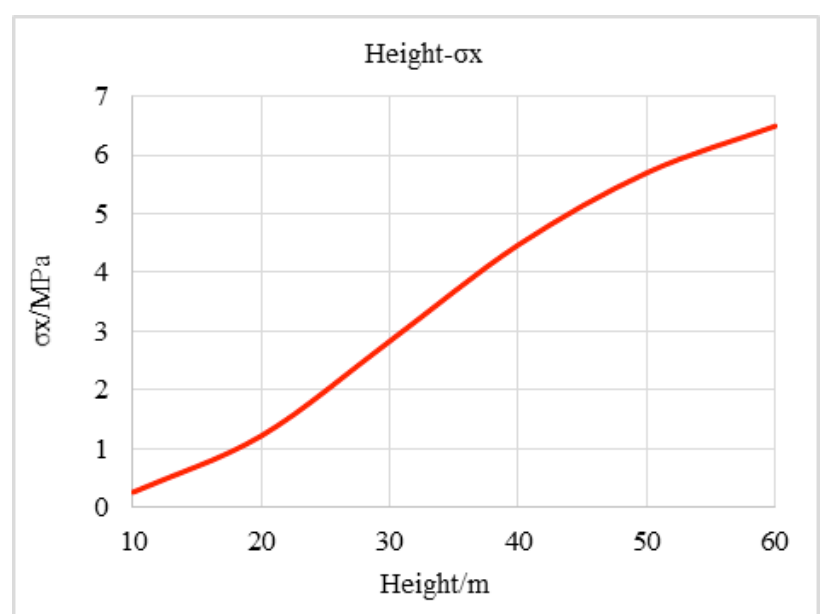

Fig.13. Relationship between induced stress and fracture height in the minimum principal stress direction 


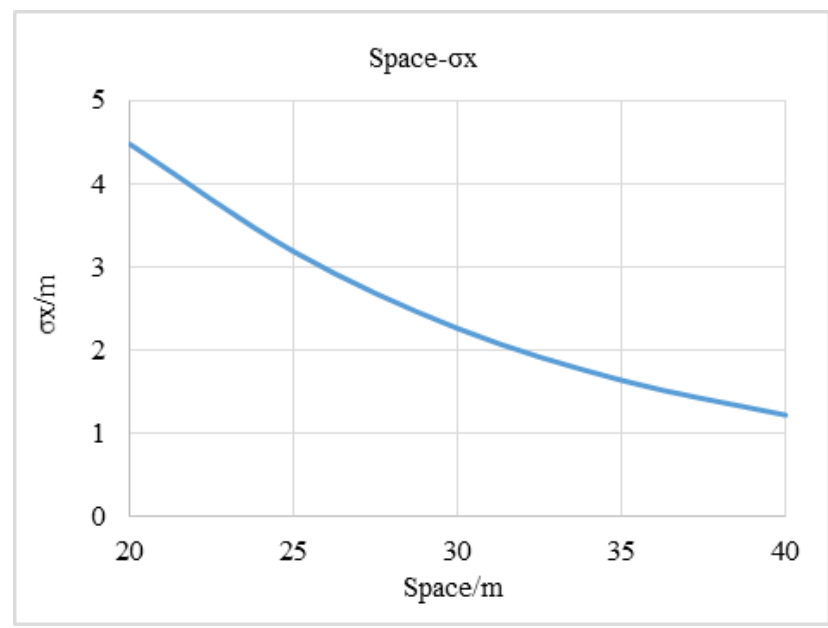

Fig.14. Relationship between induced stress and fracture interval in the minimum principal stress direction

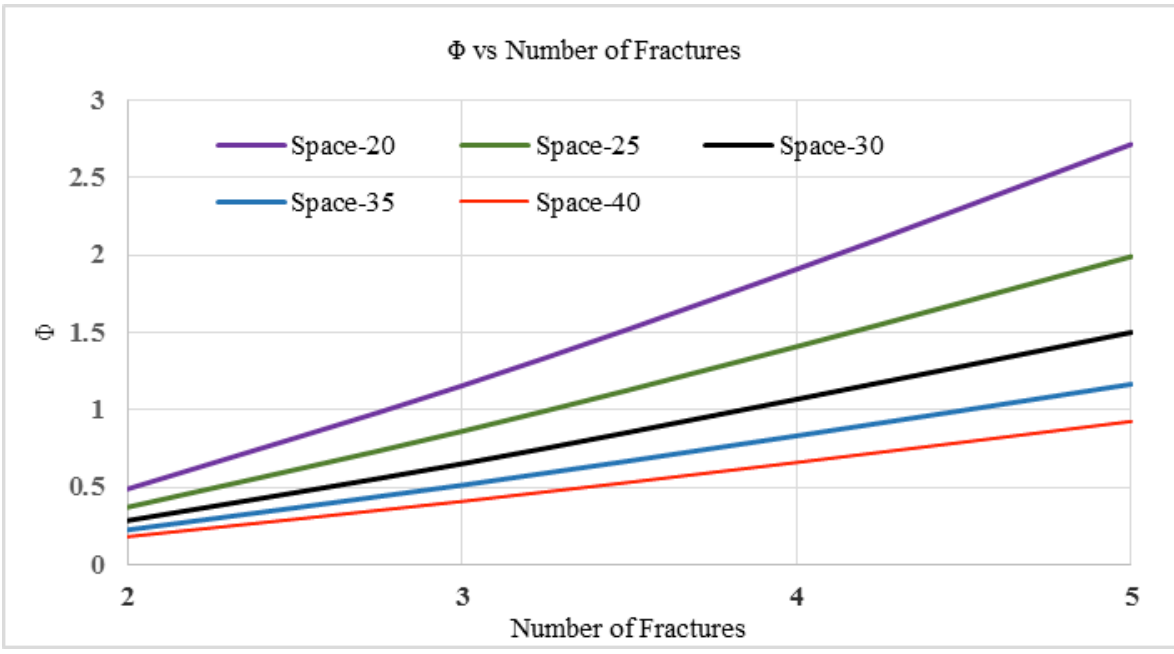

Fig.15. Relationship between induced stress correction factor and fracture number

\subsection{Relationship between induced stress and dimensionless fracture space}

The mathematical description of ISCF was presented in Section III, and the key point is to simplify original mathematical model through dimensionless fracture space. This paper analyzes the relationship between the dimensionless fracture space and the induced stress, as shown in Fig. 16. a) With the increase of the dimensionless fracture space, the induced stress in minimum principal stress direction will increase. (2) The curve is S-shaped. When the dimensionless fracture space is small or large, the increment of the induced stress is relatively steady. In the area of $1<\mathrm{h} / \mathrm{d}<2$, the increasing induced stress improves. Thus, this area is the "golden window" for the complex fracture network. Hence, $[1,2]$ can be the reference of the ratio of half fracture height and fracture interval in fracturing design. A complex fracture can be efficiently formed through the stress interference.

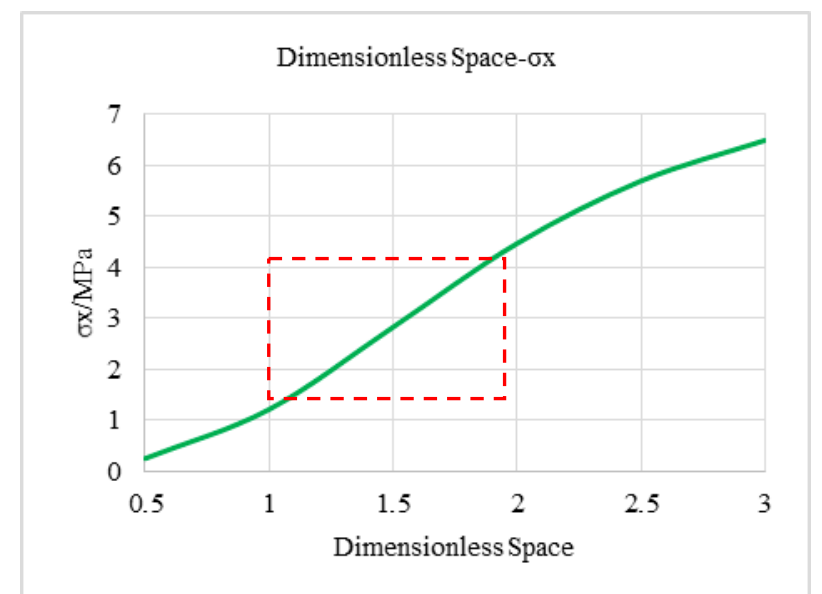

Fig.16. Relationship between induced stress and dimensionless fracture space in the minimum principal stress direction

\subsection{Numerical simulation of circumferential induced stress field}

The influence of fracture extension pressure under multifractures induced stress was discussed in Section 3.3. The results of circumferential induced stress of single and numerous fractures, as shown in Figure 15, were obtained according to the deduced numerical equation of induced stress. The blue line represents the induced stress increment when a single fracture initiates, the red line denotes the induced stress increment when numerous fractures initiate, 
and the $\mathrm{x}$ axis is the direction of the maximum principal stress. The simulation results show that a) for an open single fracture, the circumferential induced stress exhibits very good symmetry. However, for open multiple fractures, induced stress increment varies at different azimuth angles because of stress interference. b) Maximum induced stress exists at the horizontal principle stress direction, whereas minimum induced stress occurs at $30^{\circ}$ and $150^{\circ}$ with the minimum horizontal stress direction. Most of drilling direction of the horizontal wells are along the minimum horizontal principle stress. Conventional fracturing technology could benefit from this process because forming a main fracture is easily carried out. However, this process could limit shale gas fracturing because high power is required to ensure fracture propagation. Shale gas fracturing necessitates high stimulated reservoir volume and not long fracture length. Thus, the interference among fractures is not fully utilized. $30^{\circ}$ oblique cross minimum horizontal principle stress effectively creates a complex fracture.

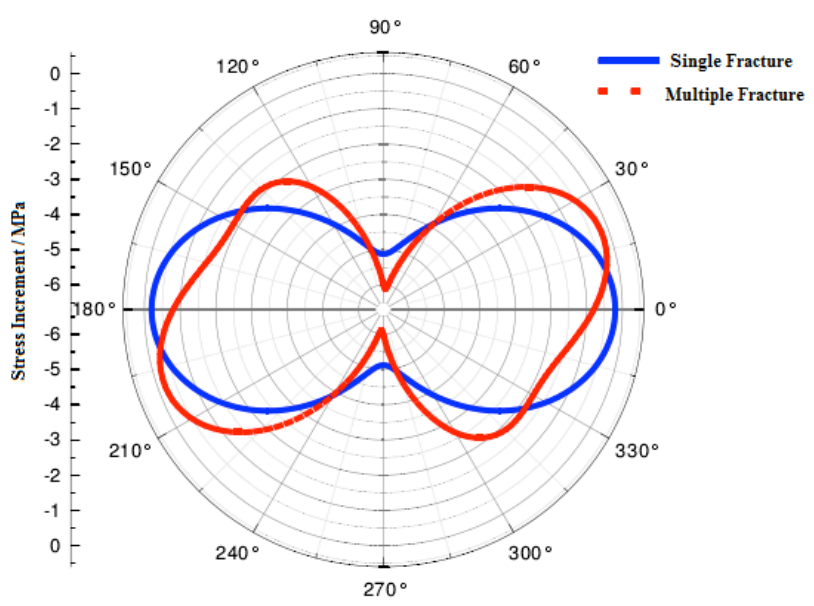

Fig.17. Numerical simulation of the induced stress field

\section{Conclusion}

To address the substantial variation of structure, lithology, and reservoir properties among different blocks, the mechanism of complex fracture propagation was studied in theory. This paper initially analyzed reservoir geology and the mechanical characteristics of rocks based on core uniaxial testing and described the different initiating modes in shale reservoirs. The influence on rock initiation caused by its mechanical parameters was examined. Fracture pressure model under multi-fractures induced stress and different initiation approaches was developed based on elastic constitutive theory, combined with linear elastic fracture mechanics and using the principle of superimposed stresses. The following conclusions were drawn:

1) The main rupture mechanics of a shale reservoir was described in three modes: a) tensile failure with shale bedding planes accompanied by shear failure, b) shear failure with shale bedding planes accompanied by tensile failure, and c) shear failure. These three modes determine the fracture initiation criterion and fracture pressure prediction model.

2) ISCF was presented and deduced to simplify equation of the surrounding rock stress field. The geometry of the fracture and laying patterns directly affect the complexity degree of HF. The simulation results indicate that fracture geometry parameters are positively correlated with induced stress, whereas these parameters are negatively correlated with fracture interval and numbers. In the optimization of fracturing design, the ratio of the half fracture height or fracture interval that belongs to $[1,2]$ area can be used as reference.

3) Maximum induced stress occurs at the horizontal principle stress direction, whereas minimum induced stress occurs at $30^{\circ}$ and $150^{\circ}$ with minimum horizontal stress $30^{\circ}$ oblique cross between directions. The drilling direction of the horizontal section and minimum horizontal principle stress is proposed.

This study combined laboratory experiments and theoretical studies and presented new findings on the initiation law of complex fractures. The fracture pressure model was simplified and adapted to actual conditions. All the findings demonstrate the importance of the subsequent development of shale reservoirs. However, actual data on the on-site monitoring of fractures are limited. In future studies, combining monitoring data and this model will provide accurate results on the initiation law of complex fractures.

\section{References}

1. Sinton, J. E., "Accuracy and reliability of China's energy statistics", China Economic Review, 12(4), 2001, pp. 373-383.

2. Zou, Caineng, Dong, Dazhong, Wang, shejiao, Li, jianzhong, "Geological characteristics, formation mechanism and resource potential of shale gas in China", Petroleum Exploration and Development, 37(6), 2010, pp. 641-653

3. Jiang, Yuqiang, Dong, Dazhong, Qi, Lin, Shen, Yanfei, Jiang, Chan, "Basic features and evaluation of shale gas reservoirs", Nature Gas Industry, 30(10), 2010, pp. 7-12

4. Yun, $\mathrm{Xu}$, Zhicheng, Huang, "Study and application of pseudo three dimensional acid fractureing design software", Oil Drilling \& Production Technology, 18(1), 1996, pp. 69-75.

5. Fisher, M. K., Davidson, B. M., Goodwin, a. k., "Integrating fracture mapping technologies to optimize stimulations in Barnett Shale", In: SPE Annual Technical Conference and Exhibition, San Antonio, USA: SPE, 2002, SPE77441.

6. Warpinski, N. R., Teufel L., "Influence of geologic discontinuities on hydraulic fracture propagation", Journal of Petroleum Technology, 39(2), 1987, pp. 209-220.
7. Renshaw, C. E., "Are large differential stresses required for straight fracture propagation paths", Journal of Structural Geology, 16(6), 1994 , pp. 817-822.

8. Gu, H., Weng, X., Lund J. B., "Hydraulic fracture crossing natural fracture at non-orthogonal angles: a criterion and its validation", SPE Production \&Operations, 27(1), 2012, pp. 20-26.

9. Olson J. E., Bahorich, B., Holder J., "Examining hydraulic fracture: natural fracture interaction in hydrostone block experiments", In: SPE Hydraulic Fracturing Technology Conference, Woodlands, USA: SPE, 2012, SPE 152618

10. Renard, F., Bernard D., Desrues J., "3D imaging of fracture propagation using synchrotron X-ray microtomography", Earth and Planetary Science Letters, 286(1), 2009, pp. 285-291.

11. Xu W., Thiercelin, M. J., Walton I. C., "Characterization of hydraulically induced shale fracture network using an analytical/semi-analytical model", In: SPE Annual Technical Conference and Exhibition, New Orleans, USA: SPE, 2009, SPE 124697. 
12. Xu, W., Le Calvez J. H., Thiercelin M. J., "Characterization of hydraulically induced fracture network using treatment and microseismic data in a tight-gas sand formation: a geomechanical approach", In: SPE Tight Gas Completions Conference, San Antonio, USA: SPE, 2009, SPE125237.

13. Xu, W., Thiercelin M. J., Ganguly, U., "Wiremesh: a novel shale fracturing simulator", In: International Oil and Gas Conference and Exhibition, Beijing, China: SPE, 2010, SPE 132218.

14. Meyer, B. R., Bazan, L. W., "A discrete fracture network model for hydraulically induced fractures-theory parametric and case studies", In: SPE Hydraulic Fracturing Technology Conference, Woodlands, USA: SPE, 2011, SPE 140514.

15. Olson, J.E., Dahi, T. A., "Modeling simultaneous growth of multiple hydraulic fractures and their interaction with natural fractures", In: SPE Hydraulic Fracturing Technology Conference, Woodlands, USA: SPE, 2009, pp. 1-7
16. Hossain, M. M., Rahman, M. K., Rahman, S. S., "Hydraulic fracture initiation and propagation: roles of wellbore trajectory, perforation and stress regimes", Journal of Petroleum Science and Engineering, 27(3), 2000, pp. 129-149.

17. Hou, Bing, Chen, Mian, Li, Zhimeng, Wang, Yonghui, Diao, Ce, "Propagation area evaluation of hydraulic fracture networks in shale gas reservoirs", Petroleum Exploration and Development, 41(6), 2014, pp. 763-768

18. Larsen, B., Gudmundsson, A., Grunnaleite, I., "Effects of sedimentary interfaces on fracture pattern, linkage, and cluster formation in peritidal carbonate rocks", Marine and Petroleum Geology, 27(7), 2010, pp. 1531-1550.

19. Nordgren, R. P., "Propagation of a vertical hydraulic fracture", SPE Journal, 12(8), 1972, pp. 306-314.

20. Warpinski, N. R., Mayerhofer, M. J., Vincent, M. C., "Stimulating unconventional reservoirs: maximizing network growth while optimizing fracture conductivity", Journal of Canadian Petroleum Technology, 48(10) 2009, pp. 39-47. 